\title{
Addressing Challenges in Ultrastructural TEM Imaging of Chlorophyceae (Green Algae)
}

\author{
Alice Dohnalkova ${ }^{1}$ and Zoe Cardon ${ }^{2}$ \\ 1. Environmental Molecular Sciences Laboratory, Pacific Northwest National Laboratory, Richland, \\ WA, USA \\ 2. Ecosystems Center, Marine Biological Laboratory, Woods Hole, MA 02543 USA
}

The class of Chlorophyceae (green algae) is extremely diverse - its families have been isolated from diametric settings spanning from desert crusts to aquatic environments. To classify and analyze their phylogenetic relationship, fine morphological data are critical for elucidation their function and processes such as cell division and desiccation tolerance. Transmission electron microcopy (TEM) was used as a valuable tool for fine ultrastructural imaging and analysis of the main cell features and organelles, as the 'fine structure' provided many vital keys to the understanding of the interrelationships and phylogeny of the algae.

In our experimental setup, diverse members of Scenedesmaceae family of green microalgae isolated from diverse environments were comparatively investigated for mechanisms of desiccation tolerance and multicellularity. Cells were analyzed by DNA sequencing, and imaged by time-lapse confocal microscopy coupled with TEM. We used high pressure freezing and freeze substitution methods for obtaining resin-embedded cells.

We found out that the desert and aquatic species all divide by multiple - rather than binary - fission, and some multicellular progeny were joined in a sheet-like coenobium (Fig 1A). Algae localized Golgi apparatus near nuclei during division. Differential desiccation tolerance across species, assessed from photosynthetic efficiency during desiccation/rehydration cycles, was accompanied by differential accumulation of intracellular reactive oxygen species (ROS) detected using a ROS-sensitive dye [2].

During our initial TEM imaging, we found out that thick cell membranes and an intricate internal morphology of photosynthetic apparatus and cellular components posed serious problems with the sample processing. Numerous artifacts were recognized as an infiltration impedance, resulting in reevaluating our traditional approach in the freeze-substitution processing. At this time, algal cells were gently pelleted, and loaded to the specimen carriers of the high pressure freezer (Leica), with as minimal moisture content as possible, ideally in a paste-like consistency. The HP-frozen pellets were processed in the automatic freeze substitution (AFS) system (S9E, Leica), following a customized protocol, but with increased infiltration times. Shaking, adapted from McDonald [1] was applied on both AFS and resin embedding steps. To allow the solutions in the AFS to mix, we used an in-house made gadget coupled to the AFS instrument that provided shaking during the entire substitution. Briefly, acetonebased solutions of $1 \%$ glutaraldehyde with $0.1 \%$ tannic acid and $1 \%$ OsO4 were used for AFS, followed by gradual infiltration in Spurr's resin on a $110 \mathrm{rpm}$ shaker for $72 \mathrm{hrs}$, and polymerization in $60 \mathrm{C}$ for 48 hrs. Ultrathin sections were prepared using Diatome 45 deg diamond knife on a Reichert UCT ultramicrotome, and post-stained with uranyl acetate alternative (Ted Pella) and lead citrate. The imaging was done using Tecnai T-12 (FEI).

We consider the increased infiltration times and shaking the main factors for the cell ultrastructure improvement. Algal chloroplasts were preserved with their finely aligned thylakoid membranes (Fig. 
1C), and numerous cellular granules and vacuoles showed consistent infiltration. Nevertheless, some organelles such as pyrenoids still experienced uneven section thickness resulting in a 'wavy' appearance (Fig1B -arrows). We assume this was due to the uneven density in the lipid - carbohydrate gradient of the cellular material.

This procedure was also successfully used for processing a variety of other algal and bacterial cells with complicated cellular compartments: green algae (Chlamydomona sp.), cyanobacteria (Rhodobacter sp., Cyanothece sp.), and yeast (Yarrowia lipolytica).

\section{References:}

[1] McDonald, K.L., Rapid Embedding Methods into Epoxy and LR White Resins for Morphological and Immunological Analysis of Cryofixed Biological Specimens. Microscopy and Microanalysis, (2014), 20(1): p. 152-163.

[2] Cardon Z., Peredo E., Dohnalkova A., Gershone H., and Bezanilla M. A model suite of green algae within the Scenedesmaceae for investigating contrasting desiccation tolerance and morphology. Journal of Cell Science (2018) - accepted Feb 2018.

[3] A part of this research was performed at the Environmental Molecular Sciences Laboratory (EMSL), a national scientific user facility, located at PNNL.
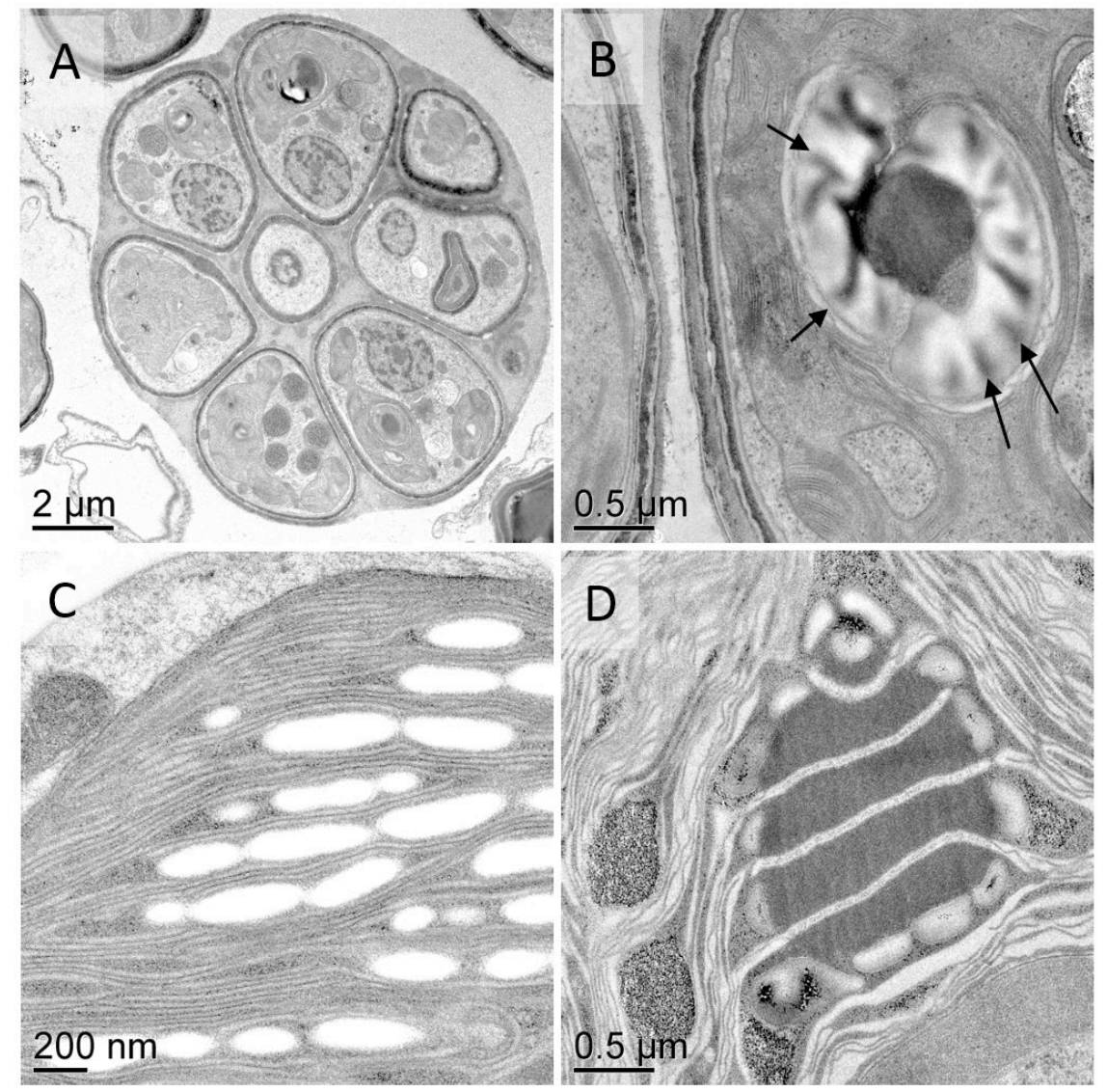

Figure 1. (A) Coenobia of divided daughter cells, often embedded in a mucilaginous matrix. (B) A pyrenoid showing a 'wavy' texture. (C) Chloroplast with aligned thylakoids and glycogen granules. (D) A pyrenoid intertwined with membranes, typical for a different family. 